\title{
Resistance Exercise Improves Spatial Learning Ability Through Phosphorylation of 5-Adenosine Monophosphate-Activated Protein Kinase in Parkinson Disease Mice
}

\author{
Sang-Hoon Kim ${ }^{1,2}$, Lakkyong Hwang ${ }^{2}$, Jun-Jang Jin ${ }^{2}$, Il-Gyu Ko ${ }^{2}$, Yong Bog Kim ${ }^{3}$, Hye-Sun Yoon ${ }^{4}$, Seung-Soo Baek ${ }^{1}$ \\ ${ }^{1}$ Department of Sport \& Health Sciences, College of Art \& Culture, Sangmyung University, Seoul, Korea \\ ${ }^{2}$ Department of Physiology, College of Medicine, Kyung Hee University, Seoul, Korea \\ ${ }^{3}$ Department of Medicine, Graduate School, Kyung Hee University, Seoul, Korea \\ ${ }^{4}$ Department of Pediatrics, Eulji Hospital, Eulji University School of Medicine, Seoul, Korea
}

Purpose: Exercise is a representative noninvasive treatment that can be applied to various diseases. We studied the effect of resistance exercise on motor function and spatial learning ability in Parkinson disease (PD) mice.

Methods: The rotarod test and beam walking test were conducted to evaluate the effect of resistance exercise on motor function, and the Morris water maze test was conducted to examine the effect of resistance exercise on spatial learning ability. The effect of resistance exercise on brain-derived neurotrophic factor (BDNF) and tropomyosin receptor kinase $\mathrm{B}$ (TrkB) expression and 5'-adenosine monophosphate-activated protein kinase (AMPK) phosphorylation was investigated by Western blot analysis. New cell generation was confirmed by immunohistochemistry for 5-bromo-2'-deoxyuridine.

Results: Resistance exercise improved coordination, balance, and spatial learning ability in PD mice. Resistance exercise enhanced new cell production, BDNF and TrkB expression, and AMPK phosphorylation in PD mice. The effect of such resistance exercise was similar to that of levodopa application.

Conclusions: In PD-induced mice, resistance exercise enhanced AMPK phosphorylation to increase BDNF expression and new neuron generation, thereby improving spatial learning ability. Resistance exercise is believed to help improve symptoms of PD.

Keywords: Resistance exercise; Parkinson disease; Spatial learning ability; Brain-derived neurotrophic factor; 5'-Adenosine monophosphate-activated protein kinase

- Fund/Grant Support: This work was supported by the Ministry of Education of the Republic of Korea and the National Research Foundation of Korea (NRF-2018S1A5A2A01036668).

- Research Ethics: The experimental procedure was approved by the Kyung Hee University Animal Ethics Committee and received approval number KHUASP (SE)-18-15.

- Conflict of Interest: No potential conflict of interest relevant to this article was reported.

\footnotetext{
- HIGHLIGHTS

- Resistance exercise improved coordination, balance, and spatial learning ability in PD mice.

- Resistance exercise enhanced new cell production, BDNF and TrkB expression, and AMPK phosphorylation in PD mice.

- The effect of such resistance exercise was similar to that levodopa application.
}

Corresponding author: Seung-Soo Baek (iD https://orcid.org/0000-0002-1340-2098 Department of Sport \& Health Care, College of Art \& Culture, Sangmyung University, 20 Hongjimun 2-gil, Jongno-gu, Seoul 03016, Korea Email: ssoop@smu.ac.kr

Submitted: September 5, 2021 / Accepted after revision: October 20, 2021
This is an Open Access article distributed under the terms of the Creative Commons Attribution Non-Commercial License (http://creativecommons.org/licenses/by-nc/4.0/) which permits unrestricted non-commercial use, distribution, and reproduction in any medium, provided the original work is properly cited. 


\section{INTRODUCTION}

The symptoms of Parkinson disease (PD) are largely divided into impairment of motor function, functional loss of autonomic nervous system, and dysfunction of nonmotor function, and this disorder causes slow movement, tremors, balance problems, and muscle stiffness [1]. From a pathophysiological point of view, dopaminergic neurons present in the substantia nigra are destroyed, and thus dopamine reduction in the striatum is characterized [2]. The cause of dopaminergic apoptosis in the substantia nigra of PD has been reported to be related to increased oxidative stress, mitochondrial dysfunction, and excitotoxicity [3,4], but a clear cause has not been elucidated.

In addition to levodopa, anticholinergic drugs that reduce tremor or drugs that inhibit type B monoamine oxidase are mainly used for the treatment of PD [5]. However, in the case of drug treatment, side effects due to long-term use and dose increase occur frequently, and peripheral disorders such as urination and defecation disorders, and central nervous system disorders such as cognitive dysfunction and delirium have been reported [6,7]. Therefore, for the effective management of PD, it is necessary to establish a treatment plan with few side effects based on the pathophysiological mechanism.

Exercise is a representative noninvasive treatment that can be applied to various diseases. Exercise has a beneficial effect on brain health along with improving cognitive function $[8,9]$. It was reported that cognitive function improvement through exercise was more effective in the old people [10,11]. Also, regular exercise improved degenerative brain diseases such as stroke, Alzheimer disease, and PD [12,13]. Exercise increased hippocampal size, blood flow, and synaptic plasticity, and neurogenesis $[9,12,14]$. Although the therapeutic effect of exercise in PD patients is widely known, aerobic exercise is mainly performed rather than resistance exercise due to several limitations. However, resistance exercise is essential to decrease the risk of falls, and it is known that resistance training in PD patients showed improvement in various physical functions [15].

In the current experiment, the effect of resistance exercise on motor function and spatial learning ability in PD-induced mice was studied. The rotarod test and beam walking test were conducted to clarify the effect of resistance exercise on motor function, and the Morris water maze test was conducted to examine the effect of resistance exercise on spatial learning ability. Western blot analysis was done to evaluate the effect of resistance exercise on the brain-derived neurotrophic factor (BDNF) and tropomyosin receptor kinase B (TrkB) expression and 5'-adenosine monophosphate-activated protein kinase (AMPK) phosphorylation. New cell generation was confirmed by immunohistochemistry for 5-bromo-2'-deoxyuridine (BrdU).

\section{MATERIALS AND METHODS}

\section{Animals and Grouping}

Male ICR mice weighing $30 \pm 2 \mathrm{~g}$ (10 weeks old) were obtained and prepared for this experiment. The entire process was confirmed by the Animal Ethics Committee of Kyung Hee University, and the following approval number was obtained: KHUSASP (SE)-18-15. The mice were classified into control group, PD group, $\mathrm{PD}$ with resistance exercise group, and PD with levodopa group.

\section{PD Induction}

PD induction was done as previously explained method [16]. The mice were intraperitoneally injected with $250 \mathrm{mg} / \mathrm{kg}$ probenecid diluted in dimethyl sulfoxide (Sigma-Aldrich Chemical Co., St. Louis, MO, USA). After 30 minutes injection of probenecid, 20-mg/kg 1-methyl-4-phenyl-1,2,3,6-tetrahydropyridine (MPTP, Sigma-Aldrich Chemical Co.) diluted in $0.9 \%$ saline was administered subcutaneously using a $100-\mu \mathrm{L}$ syringe. All groups except the control group were administered MPTP 10 times during 5 weeks.

\section{Resistance Exercise and Drug Application}

The resistance exercise was conducted as previously explained method $[16,17]$. From the 7 th day after MPTP injection, the mice in the exercise group were given ladder-climbing exercise 5 days a week during 5 weeks. The mice were allowed to climb ladder with $30 \%$ of their weight on their tails in the first week, $40 \%$ of their weight on their tails at $2-3$ weeks, and $50 \%$ of their weight on their tails at 4-5 weeks. The mice in the PD with levodopa group were orally administered with $25-\mathrm{mg} / \mathrm{kg}$ levodopa $(100 \mu \mathrm{L}), 5$ days a week during 5 weeks.

\section{Rotarod Test}

The rotarod test was performed on the 34th day of the first MPTP administration as previously explained method $[18,19]$. The experimental animals were placed on a rotating cylinder of a rotarod equipment and started at a speed of $4 \mathrm{rpm}$. Thereafter, the speed of about $4 \mathrm{rpm}$ was gradually increased to reach 45 rpm at 300 seconds, which is the end time of the experiment. 
The latency until the experimental animals fell from the rotating rotarod device was measured.

\section{Beam Walking Test}

A beam walking test was performed on the 35th day of the first MPTP administration as previously explained method [18]. The experimental animals were allowed to walk horizontally on an acrylic rod having a width of $1 \mathrm{~cm}$ and a length of $100 \mathrm{~cm}$, and the time it took to move without falling to the end point $(100 \mathrm{~cm})$ was measured.

\section{Morris Water Maze Test}

Morris water maze test was done as previously explained method $[20,21]$. For this evaluation, learning started on the 37th day of the first MPTP administration, followed by a 4-day learning period, and then the main test was conducted on the 41st day. A circular pool with a diameter of $140 \mathrm{~cm}$ and a height of 45 $\mathrm{cm}$ was filled with water $\left(28^{\circ} \mathrm{C} \pm 2^{\circ} \mathrm{C}\right)$, and paint of a color symmetrical to the experimental animals was dissolved in water. After training the mice to move from the initial starting point to the destination (diameter, $15 \mathrm{~cm}$; height, $35 \mathrm{~cm}$ ), when all learning was completed, the destination was removed and the waiting time in the destination area was measured. In all procedures, the movement of the experimental animals was quantified using a Smart Video Tracking System (Smart version 2.5, Panlab, Barcelona, Spain).

\section{Tissue Preparation}

After the Morris water maze test was completed, all mice were anesthetized with Zoletil 50 (10 mg/kg, Vibac Laboratories, Carros, France) after which the mice were sacrificed. For fixation in mice, $50 \mathrm{mM}$ phosphate-buffered saline and $4 \%$ paraformaldehyde were injected via the heart. After brain removal, $40-\mu \mathrm{m}$-thick coronal sections were made using a freezing microtome (Leica, Nussloch, Germany).

\section{Western Blotting}

Western blot analysis for BDNF, TrkB, and AMPK was done as previously explained method [14,22]. Lysis buffer was applied to lyse hippocampal tissues, and protein content was detected by a colorimetric protein assay kit (Bio-Rad, Hercules, CA, USA). Sodium dodecyl sulfate-polyacrylamide gel was used to separated 30- $\mu$ g protein, then reaction mixture was transferred to a nitrocellulose membrane, stopped reaction by applying dehydrated milk, and then incubated by primary antibodies. Mouse anti- $\beta$ actin (1:3,000; Santa Cruz Biotechnology, Santa Cruz, CA, USA), rabbit anti-BDNF (1:1,000; Santa Cruz Biotechnology), rabbit anti-TrkB (1:1,000; Santa Cruz Biotechnology), and rabbit anti-AMPK (1:1,000; Santa Cruz Biotechnology) were selected as the primary antibodies. Horseradish peroxidaseconjugated secondary antibodies were used, and enhanced chemiluminescence detection system (Santa Cruz Biotechnology) measured the expression of bands.

\section{Immunohistochemistry}

$\mathrm{BrdU}$ immunohistochemistry was done as previous explained method $[14,22]$. BrdU-specific mouse monoclonal antibody (1:600; Roche, Mannheim, Germany) was applied to the sections overnight at $4^{\circ} \mathrm{C}$. Biotinylated mouse secondary antibody (1:200; Vector Laboratories, Burlingame, CA, USA) was treated to the sections for 1 hour and $\mathrm{ABC}$ complex (1:100; Vector Laboratories) was applied for 1 hour. In order to visualize, the sections were treated to $50 \mathrm{mM}$ Tris- $\mathrm{HCl}(\mathrm{pH}, 7.6)$ with $0.03 \%$ diaminobenzidine, then $0.03 \% \mathrm{H}_{2} \mathrm{O}_{2}$, and $40-\mathrm{mg} / \mathrm{mL}$ nickel chloride for 5 minutes.

\section{Data Analysis}

The number of BrdU-positive cells in the hippocampal dentate gyrus was measured from the hemilateral side and presented as the cell number $/ \mathrm{mm}^{2}$. The detected bands from the Western blot were counted using a densitometer. For data analysis, 1-way analysis of variance and Duncan post hoc test was conducted and the results were presented as mean \pm standard error of the mean. $\mathrm{P}<0.05$ showed statistical significance.

\section{RESULTS}

\section{Coordination and Balance}

The induction of PD reduced latency in rotarod test and increased arrival time in beam walking test compared to the control group. Meanwhile, resistance exercise extended waiting time in rotarod test and reduced arrival time in beam walking test, and had a similar effect to the group treated with levodopa (Fig. 1).

\section{Spatial Learning Ability}

The induction of $\mathrm{PD}$ shortened the time spent at the target zone compared to the control group. Meanwhile, resistance exercise lengthened the time spent at the target zone, and had a similar effect to the group treated with levodopa (Fig. 2). 

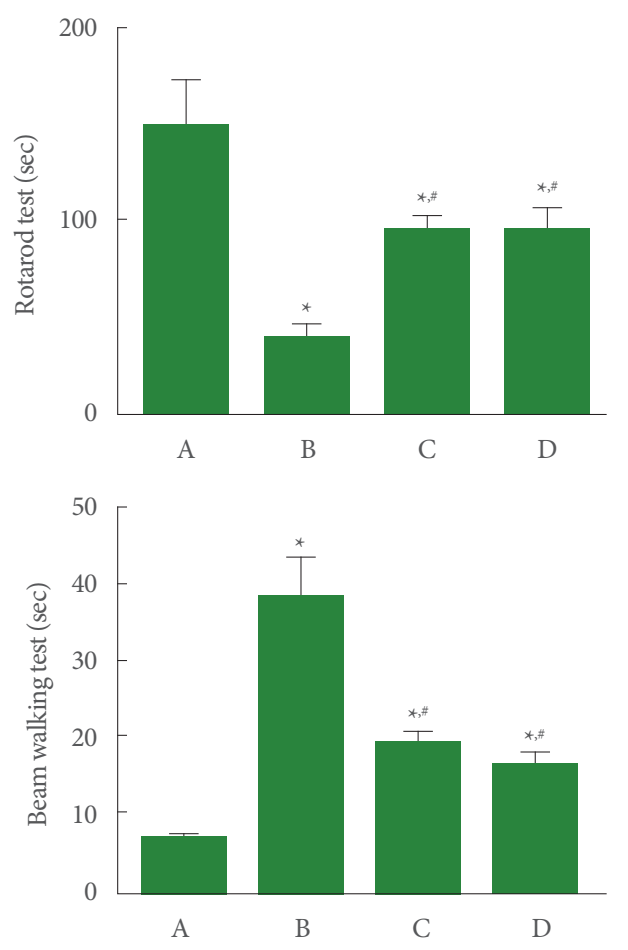

Fig. 1. Effect of resistance exercise on motor function. Upper panel: rotarod test. Lower panel: beam walking test. A, control group; B, Parkinson disease (PD) group; C, PD with resistance exercise group; $\mathrm{D}, \mathrm{PD}$ with levodopa group. ${ }^{\star} \mathrm{P}<0.05$ compared to the control group. ${ }^{*} \mathrm{P}<0.05$ compared to the $\mathrm{PD}$ group.

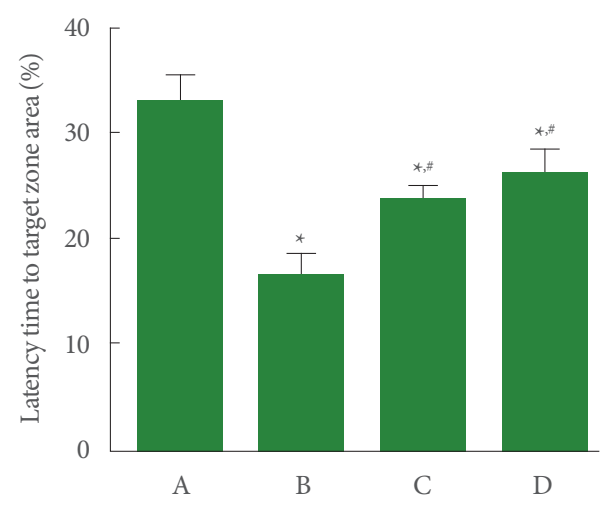

Fig. 2. Effect of resistance exercise on spatial learning memory in Morris water maze test. A, control group; B, Parkinson disease (PD) group; C, $\mathrm{PD}$ with resistance exercise group; $\mathrm{D}, \mathrm{PD}$ with levodopa group. ${ }^{\star} \mathrm{P}<0.05$ compared to the control group. ${ }^{*} \mathrm{P}<0.05$ compared to the $\mathrm{PD}$ group.

\section{New Cell Generation}

The induction of PD suppressed the number of BrdU-positive cells in the hippocampal dentate gyrus compared to the control group. Meanwhile, resistance exercise potentiated the number
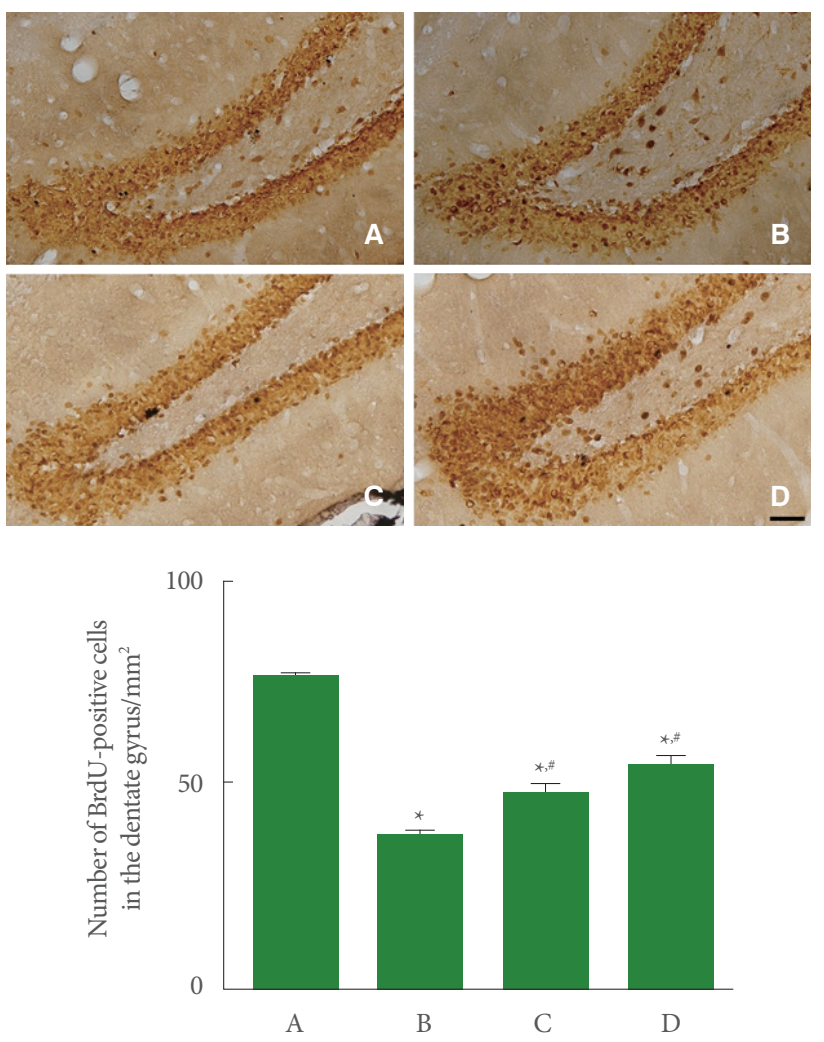

Fig. 3. Effect of resistance exercise on cell proliferation in the hippocampus. Upper panel: photomicrographs of 5-bromo-2'deoxyuridine (BrdU)-positive cells. The scale bar represents 150 $\mu \mathrm{m}$. Lower panel: number of BrdU-positive cells in each group. A, control group; B, Parkinson disease (PD) group; C, PD with resistance exercise group; $\mathrm{D}, \mathrm{PD}$ with levodopa group. ${ }^{\star} \mathrm{P}<0.05$ compared to the control group. ${ }^{*} \mathrm{P}<0.05$ compared to the $\mathrm{PD}$ group.

of BrdU-positive cells, and had a similar effect to the group treated with levodopa (Fig. 3).

\section{BDNF and TrkB Expression}

The induction of PD reduced BDNF and TrkB expression in the hippocampus compared to the control group. Meanwhile, resistance exercise increased BDNF and TrkB expression, and had a similar effect to the group treated with levodopa (Fig. 4).

\section{AMPK Phosphorylation}

The induction of PD reduced the ratio of AMPK phosphorylation in the hippocampus compared to the control group. Meanwhile, resistance exercise enhanced ratio of AMPK phosphorylation, and had a similar effect to the group treated with levodopa (Fig. 5). 

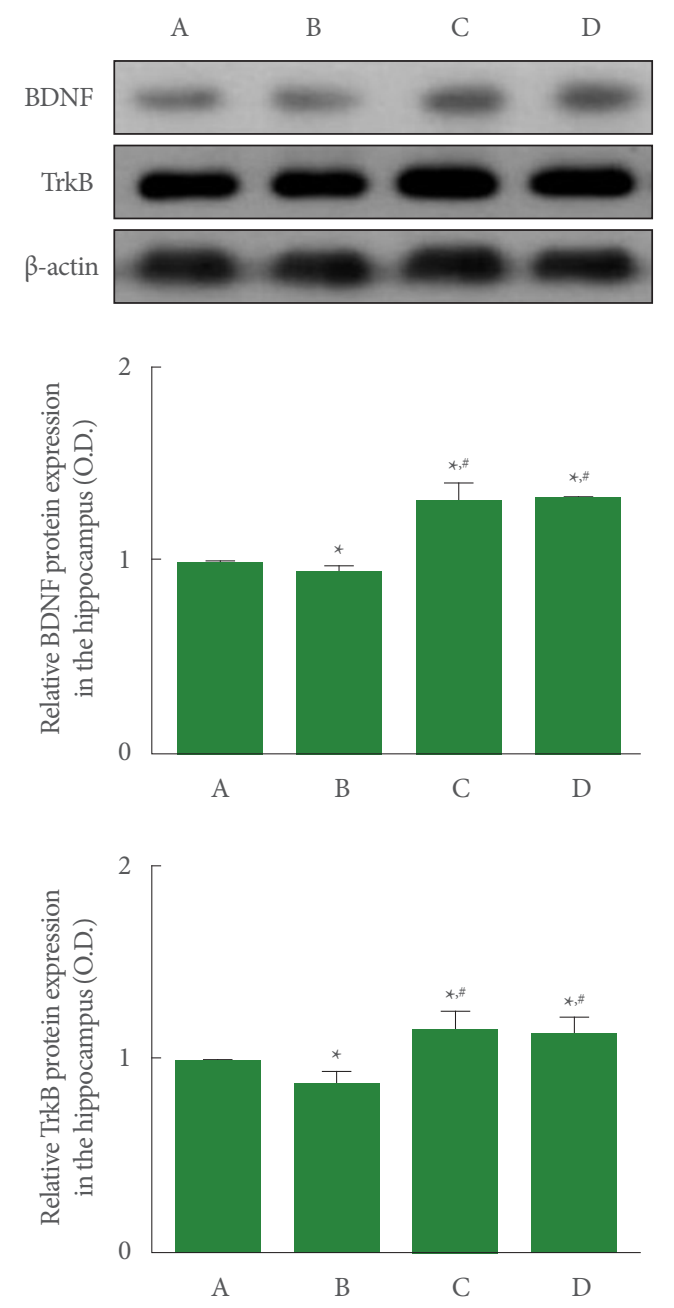

Fig. 4. Effect of resistance exercise on brain-derived neurotrophic factor (BDNF) and tropomyosin kinase B (TrkB) expression in the hippocampus. Upper panel: representative expression of BDNF and TrkB. Middle panel: relative expression of BDNF in each group. Lower panel: relative expression of TrkB in each group. A, control group; B, Parkinson disease (PD) group; C, PD with resistance exercise group; D, PD with levodopa group. ${ }^{*} \mathrm{P}<0.05$ compared to the control group. ${ }^{*} \mathrm{P}<0.05$ compared to the $\mathrm{PD}$ group.

\section{DISCUSSION}

Traumatic brain-injured rats showed obvious neurological deficits with decreased latency in the rotarod test and increased arrival time in the beam gait test [18]. MPTP-induced Parkinsonism showed motor deficits in behavioral tests including beam walking test and pole test [23]. Swimming exercise improved motor coordination and balance in the rotarod test and vertical pole test of the experimental autoimmune encephalomyelitis

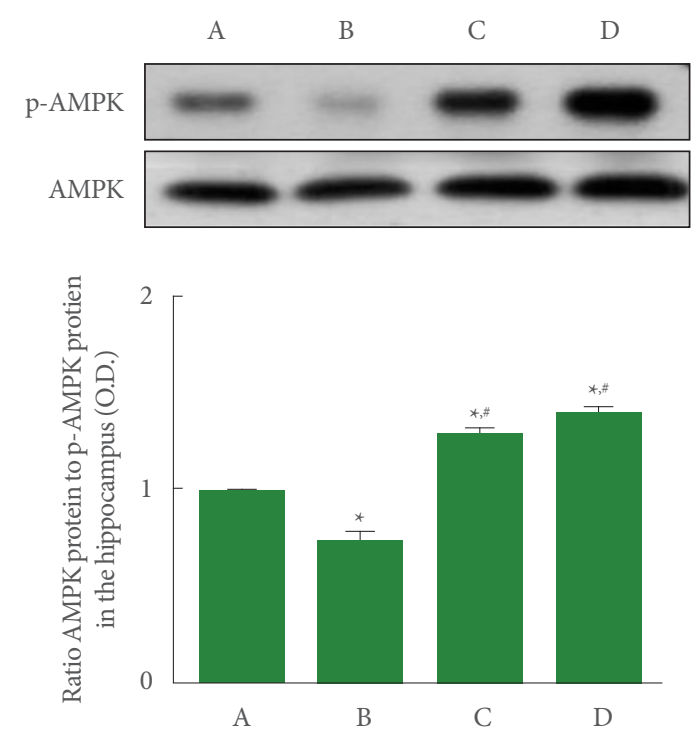

Fig. 5. Effect of resistance exercise on phosphorylation of adenosine monophosphate protein kinase (AMPK) in the hippocampus. Upper panel: representative expression of AMPK. Lower panel: ratio of AMPK to phosphorylated AMPK (pAMPK) in each group. A, control group; B, Parkinson disease (PD) group; C, $\mathrm{PD}$ with resistance exercise group; $\mathrm{D}, \mathrm{PD}$ with levodopa group. ${ }^{\star} \mathrm{P}<0.05$ compared to the control group. ${ }^{*} \mathrm{P}<$ 0.05 compared to the $\mathrm{PD}$ group.

rats [19]. Treadmill running improved motor function in the rotarod test and foot fault test of the photothrombotic stroke mice [24]. In the results of this experiment, the induction of PD decreased coordination and balance in the rotarod test and beam walking test compared to the control group. However, resistance exercise improved coordination and balance, and the effect was similar to that of levodopa treatment.

MPTP-induced PD mice showed impairment of spatial learning ability in the Morris water maze test with tyrosine hydroxylase-positive neuronal loss [25]. Impairment of motor skills in PD patients is associated with cognitive impairment. PD patients with mild cognitive impairment performed significantly worse in accuracy, dexterity, and speed (arm hand movements) compared to the PD patients without mild cognitive impairment [26]. Treadmill running improved short-term memory in step-through avoidance task in the photothrombotic stroke mice [24]. In the results of this experiment, the induction of PD impaired spatial learning ability in Morris water maze test compared to the control group. However, resistance exercise improved spatial learning ability, and the effect was similar to that of levodopa treatment.

There are many reports that exercise enhances newly gener- 
ated neurons in the hippocampus. Treadmill running enhanced new cell production in the hippocampal dentate gyrus of oldaged rats [11]. Reduced hippocampal neurogenesis was observed in the 6-hydroxydopamine-induced PD rat model [27]. Treadmill running increased short-term memory by enhancing the production of new neurons in rat pups born to obese mother rats [14]. Treadmill running exerted a potentiating effect on synaptic plasticity and new cell generation in the hippocampus of stroke mice [24]. In the results of this experiment, the induction of PD inhibited new cell formation in the hippocampal dentate gyrus compared to the control group. However, resistance exercise potentiated new cell production, and the effect was similar to that of levodopa treatment.

BDNF and its receptor TrkB, which are most abundantly expressed in the brain, modulate axonal and dendritic growth in the nervous system, and they play important roles in learning and memory process [28]. Dysfunction of striatal neurons is a major cause of motor impairment in Huntington's disease, and BDNF supports the promotion of survival, maturation, and connectivity of striatal neurons during brain development [29]. Treadmill running enhanced BDNF and TrkB expression in the hippocampus of rat pups born to obese maternal rats [14]. In mice with photothrombotic stroke, a potentiating effect of treadmill running on hippocampal neuronal production by increasing hippocampal BDNF and $\operatorname{TrkB}$ expression was shown [24]. Treadmill running ameliorated short-term and spatial working memory impairment though increment of BDNF and TrkB expression in socially isolated rats [30]. In the results of this experiment, the induction of PD suppressed the expression of BDNF and TrkB in the hippocampus compared to the control group. However, resistance exercise increased expression of BDNF and TrkB, and the effect was similar to that of levodopa treatment.

AMPK is a heterotrimeric protein complex made by $\alpha, \beta$, and $\gamma$ subunits and is the serine/threonine kinase that modulates cellular energy homeostasis. When the a subunit of AMPK is phosphorylated, AMPK is activated [31]. In exercising muscle cells, AMPK increased blood supply by stimulating angiogenesis [32]. AMPK activation produced an antidepressant effect, which was potentially mediated by hippocampal neurogenesis via $B D N F / T r k B$ signaling in neurons [33]. Increased AMPK phosphorylation by voluntary wheel running in aged rats improved vascular endothelial growth factor expression and alleviated sarcopenia [34]. In the results of this experiment, the induction of PD suppressed AMPK phosphorylation in the hip- pocampus compared to the control group. However, resistance exercise enhanced AMPK phosphorylation, and the effect was similar to that of levodopa treatment.

In the current study, resistance exercise in PD-induced mice enhanced AMPK phosphorylation to increase BDNF expression and new neuron generation, thereby improving spatial learning ability. Resistance exercise is believed to help improve symptoms of PD.

\section{AUTHOR CONTRIBUTION STATEMENT}

- Conceptualization: $S H K$

- Data curation: LH, JJJ, IGK

- Formal analysis: $L H$, JJJ, IGK

- Funding acquisition: $S S B$

- Methodology: LH, JJJ, IGK

- Project administration: SSB

- Visualization: YBK, HSY

-Writing-original draft: $S H K$

-Writing-review \& editing: SHK

\section{ORCID}

$\begin{array}{ll}\text { Sang-Hoon Kim } & 0000-0002-8867-8822 \\ \text { Lakkyong Hwang } & 0000-0002-7916-3229 \\ \text { Jun-Jang Jin } & 0000-0001-5594-5915 \\ \text { Il-Gyu Ko } & 0000-0003-2696-6869 \\ \text { Yong Bog Kim } & 0000-0001-5448-5830 \\ \text { Hye-Sun Yoon } & 0000-0002-8409-2963 \\ \text { Seung-Soo Baek } & 0000-0002-1340-2098\end{array}$

\section{REFERENCES}

1. Jankovic J. Parkinson's disease: clinical features and diagnosis. J Neurol Neurosurg Psychiatry 2008;79:368-76.

2. Wong SS, Li RH, Stadlin A. Oxidative stress induced by MPTP and MPP+: selective vulnerability of cultured mouse astrocytes. Brain Res 1999;836:237-44.

3. Mariani E, Polidori MC, Cherubini A, Mecocci P. Oxidative stress in brain aging, neurodegenerative and vascular diseases: an overview. J Chromatogr B Analyt Technol Biomed Life Sci 2005;827:6575.

4. Blandini F. An update on the potential role of excitotoxicity in the pathogenesis of Parkinson's disease. Funct Neurol 2010;25:65-71.

5. Dezsi L, Vecsei L. Monoamine oxidase B inhibitors in Parkinson's 
disease. CNS Neurol Disord Drug Targets 2017;16:425-39.

6. Jankovic J, Aguilar LG. Current approaches to the treatment of Parkinson's disease. Neuropsychiatr Dis Treat 2008;4:743-57.

7. Ebersbach G, Ip CW, Klebe S, Koschel J, Lorenzl S, Schrader C, et al. Management of delirium in Parkinson's disease. J Neural Transm (Vienna) 2019;126:905-12.

8. van Praag H. Exercise and the brain: something to chew on. Trends Neurosci 2009;32:283-90.

9. Mattson MP. Energy intake and exercise as determinants of brain health and vulnerability to injury and disease. Cell Metab 2012;16: 706-22.

10. Colcombe S, Kramer AF. Fitness effects on the cognitive function of older adults: a meta-analytic study. Psychol Sci 2003;14:125-30.

11. Kim SE, Ko IG, Kim BK, Shin MS, Cho S, Kim CJ, et al. Treadmill exercise prevents aging-induced failure of memory through an increase in neurogenesis and suppression of apoptosis in rat hippocampus. Exp Gerontol 2010;45:357-65.

12. Baek SS. Role of exercise on the brain. J Exerc Rehabil 2016;12:3805.

13. Baek SS, Kim SH. Treadmill exercise ameliorates symptoms of Alzheimer disease through suppressing microglial activation-induced apoptosis in rats. J Exerc Rehabil 2016;12:526-34.

14. Ji ES, Kim YM, Ko YJ, Baek SS. Treadmill exercise in obese maternal rats during pregnancy improves short-term memory through neurogenesis in the hippocampus of rat pups. J Exer Rehabil 2020; 16:392-7.

15. Leal LC, Abrahin O, Rodrigues RP, da Silva MC, Araújo AP, de Sousa EC, et al. Low-volume resistance training improves the functional capacity of older individuals with Parkinson's disease. Geriatr Gerontol Int 2019;19:635-40.

16. Kim SH, Ko YJ, Baek SS. Resistance exercise improves short-term memory through inactivation of NF- $\mathrm{kB}$ pathway in mice with Parkinson disease. J Exer Rehabil 2021;17:81-7.

17. Kim J. Low-intensity tower climbing resistance exercise reduces experiาmentally induced atopic dermatitis in mice. J Exer Rehabil 2019;15:518-25.

18. Tang R, Lin YM, Liu HX, Wang ES. Neuroprotective effect of docosahexaenoic acid in rat traumatic brain injury model via regulation of TLR4/NF-Kappa B signaling pathway. Int J Biochem Cell Biol 2018;99:64-71.

19. Kim JY, Yi ES, Lee H, Kim JS, Jee YS, Kim SE, et al. Swimming exercise ameliorates symptoms of MOG-induced experimental autoimmune encephalomyelitis by inhibiting inflammation and demyelination in rats. Int Neurourol J 2020;24(Suppl 1):S39-47.

20. Park SS, Shin MS, Park HS, Kim TW, Kim CJ, Lim BV. Treadmill exercise ameliorates nicotine withdrawal-induced symptoms. J Exerc Rehabil 2019;15:383-91.

21. Ko YJ, Kim BK, Ji ES. Treadmill exercise in obese maternal rats during pregnancy improves spatial memory through activation of phosphatidylinositol 3-kinase pathway in the hippocampus of rat pups. J Exer Rehabil 2020;16:483-8.

22. Park HS, Kim TW, Park SS, Lee SJ. Swimming exercise ameliorates mood disorder and memory impairment by enhancing neurogenesis, serotonin expression, and inhibiting apoptosis in social isolation rats during adolescence. J Exerc Rehabil 2020;16:132-40.

23. Chang HC, Liu KF, Teng CJ, Lai SC, Yang SE, Ching H, et al. Sophora tomentosa extract prevents MPTP-induced Parkinsonism in C57BL/6 mice via the inhibition of GSK-3 $\beta$ phosphorylation and oxidative stress. Nutrients 2019;11:252.

24. Hong M, Kim M, Kim TW, Park SS, Kim MK, Park YH, et al. Treadmill exercise improves motor function and short-term memory by enhancing synaptic plasticity and neurogenesis in photothrombotic stroke mice. Int Neurourol J 2020;24(Suppl 1):S28-38.

25. Deguil J, Chavant F, Lafay-Chebassier C, Pérault-Pochat MC, Fauconneau B, Pain S. Neuroprotective effect of PACAP on translational control alteration and cognitive decline in MPTP parkinsonian mice. Neurotox Res 2010;17:142-55.

26. Dahdal P, Meyer A, Chaturvedi M, Nowak K, Roesch AD, Fuhr P. Fine motor function skills in patients with Parkinson disease with and without mild cognitive impairment. Dement Geriat Cogn Disord 2016;42:127-34.

27. Singh S, Mishra A, Mishra SK, Shukla S. ALCAR promote adult hippocampal neurogenesis by regulating cell-survival and cell death-related signals in rat model of Parkinson's disease like-phenotypes. Neurochem Int 2017;108:388-96.

28. Gonzalez A, Moya-Alvarado G, Gonzalez-Billaut C, Bronfman FC. Cellular and molecular mechanisms regulating neuronal growth by brain-derived neurotrophic factor. Cytoskeleton (Hoboken) 2016; 73:612- 28.

29. Baydyuk M, Xu B. BDNF signaling and survival of striatal neurons. Front Cell Neurosci 2014;8:254.

30. Kim TW, Park SS, Shin MS, Park HS, Baek SS. Treadmill exercise ameliorates social isolation-induced memory impairment by enhancing silent information regulator-1 expression in rats. J Exerc Rehabil 2020;16:227-33.

31. Jeon SM. Regulation and function of AMPK in physiology and diseases. Exp Mol Med 2016;48:e245.

32. Ouchi N, Shibata R, Walsh K. AMP-activated protein kinase signaling stimulates VEGF expression and angiogenesis in skeletal muscle. Circ Res 2005;96:838-46. 
33. Odaira T, Nakagawasai O, Takahashi K, Nemoto W, Sakuma W, Lin JR, et al. Mechanisms underpinning AMP-activated protein kinase-related effects on behavior and hippocampal neurogenesis in an animal model of depression. Neuropharmacology 2019;150:12133.
34. Ko YJ, Ko IG. Voluntary wheel running exercise improves aginginduced sarcopenia via activation of peroxisome proliferator-activated receptor gamma coactivator-1 $\alpha /$ fibronectin type III domaincontaining protein 5/adenosine monophosphate-activated protein kinase signaling pathway. Int Neurourol J 2021;25(Suppl 1):S27-34. 